\title{
Uso de sillas ajustables en docentes que realizan teletrabajo y presentan molestias osteomioarticulares en la Unidad Educativa Hispano América.
} Use of adjustable chairs in teachers who telework and present
osteomyoarticular discomfort at the Hispano America Educational Unit.

Paola Estefanía Vargas Arboleda. ${ }^{1}$ \& Manolo Alexander Córdova Suárez. ${ }^{2}$

Recibido: 11-07-2021 / Revisado: 25-07-2021 /Aceptado: 15-08-2021/ Publicado: 05-09-2021

DOI: https://doi.org/10.33262/anatomiadigital.v4i3.1.1861

\begin{abstract}
.
Resumen.

Introduction. COVID-19 has changed Introducción. El COVID-19 ha reality, forcing the population to adopt new work scenarios, among which those concerning the teaching-learning modality stand out, making telework a necessity to adapt this way of work, which has increased osteomyoarticular discomfort (AOM) due to postural static load with the use of dysergonomic chairs. Objective. This research applies the use of adjustable chairs to reduce OMA problems in teachers who telework at Hispano America Educational Unit cambiado la realidad, obligando a la población a adoptar nuevos escenarios de trabajo, entre las cuales destacan los concernientes a la modalidad de enseñanza - aprendizaje, convirtiendo al teletrabajo en una necesidad para adaptar esta manera de labores, lo que ha incrementado las molestias osteomioarticulares (OMA) por carga estática postural con el uso de sillas disergonómicas. Objetivo. Esta investigación aplica el uso de sillas

1 Universidad Regional Autónoma de los Andes, Posgradista. Ambato, Ecuador. pg.paolaeva09@uniandes.edu.ec, (i) https://orcid.org/0000-0002-7629-6376

2 Universidad Nacional de Chimborazo, Facultad de Ingeniería. Riobamba, Ecuador. manolo.cordova@unach.edu.ec, https://orcid.org/0000-0001-6786-7926
\end{abstract}


(UEHA), using the Rapid Office Strain Assessment (ROSA) method for verification. Methodology. It began with the identification of the most frequent OMA problems using the Nordic Questionnaire as the main tool. Prior to the implementation of the use of adjustable chairs, the postures adopted by the teachers who carry out teleworking of the technical commission of the UEHA accounting area were evaluated, with the ROSA method that starts from the observation of jobs in which the worker remains seated in a chair, in front of a table, and operating a computer with a data display screen. Results. From the Nordic questionnaire, it was observed that of the study population, $75 \%$ of the teachers have presented OMA symptoms such as lumbar pain. From the results of the application of the corrective measure with the use of adjustable chairs using the ROSA method, an overall decrease was obtained from the initial score of 7 , to a final ergonomic risk level for forced postures of 4; improving posture and reducing low back pain caused. Conclusion. With the use of the adjustable chair in the workplace, a reduction of three points to the ROSA final score was achieved, which results in a low risk of the workplace.

Keywords: Teachers, teleworking, osteomyoarticular discomfort, Nordic questionnaire, ROSA, adjustable chairs. ajustables, para disminuir los problemas OMA en los docentes que realizan teletrabajo en la Unidad Educativa Hispano América (UEHA), utilizando como medio de verificación el método Rapid Office Strain Assessment (ROSA). Metodología. Se inició con la identificación de los problemas OMA más frecuentes utilizando como herramienta principal el Cuestionario Nórdico. Previo a la implementación del uso de sillas ajustables se evaluó las posturas adoptadas por los docentes que realizan teletrabajo de la comisión técnica del área de contabilidad de la UEHA, con el método ROSA que parte de la observación de puestos de trabajo en los que el trabajador permanece sentado en una silla, frente a una mesa, y manejando un equipo informático con pantalla de visualización de datos. Resultados. Del cuestionario Nórdico se observó que de la población en estudio el $75 \%$ de los docentes han presentado molestias OMA como dolor lumbar. De los resultados de la aplicación de la medida correctiva con el uso de sillas ajustables utilizando el método ROSA, se obtuvo una disminución global desde la puntación inicial de 7, a un nivel de riesgo ergonómico por posturas forzadas final de 4; mejorando la postura y disminuyendo el dolor lumbar causado. Conclusión. Con el uso de la silla ajustable en el puesto de trabajo se logró una disminución del tres puntos a la puntuación final ROSA, con lo cual se obtiene un riesgo bajo del puesto de trabajo.

Palabras claves: Docentes, teletrabajo, molestias osteomioarticulares, cuestionario nórdico, ROSA, sillas ajustables. 


\section{Introducción}

El teletrabajo se ha convertido en una alternativa a la cual se han acogido múltiples empleadores como una modalidad a distancia, para romper el paradigma de que un trabajo solamente puede desarrollarse de forma presencial, con incremento significativo en el mes de Marzo de 2020 a raíz de la emergencia sanitaria provocada por el COVID - 19, obligando a los trabajadores a ejecutar sus labores desde cualquier sitio con acceso a internet y un ordenador (Montero Ulate et al., 2020).

La globalización y los avances tecnológicos han conllevado a realizar transformaciones en el ámbito social, corporativo y laboral, en toda la población mundial, pues el teletrabajo se caracteriza por el uso de las tecnologías de la información y comunicación (TICS), siendo innecesaria la presencia física del trabajador en las instalaciones de las empresas, en especial los centros educativos de segundo nivel (Chuco Aguilar, 2021).

Cabe mencionar que este nuevo sistema de trabajo tipo "home office", ha originado una nueva problemática que son las inadecuadas cargas estáticas posturales, debido a que permanecen horas sentados realizando sus respectivas actividades, ocupando espacios no adecuados para el trabajo, con sillas que no brindan un soporte adecuado a la región lumbar y que no pueden adaptarse a las necesidades de cada trabajador, en especial los docentes que ejecutan su trabajo con esta modalidad, es por ello que el 50\% de la población presenta dolores en región lumbar (Vallejo Morán et al., 2021), por lo que especialistas recomiendan el uso de sillas ajustables a fin de mitigar lesiones y dolores que pueden generarse a lo largo del teletrabajo, para así de precautelar la seguridad y salud laboral de los docentes (Benites-Morillas et al., 2021).

Con la implementación del teletrabajo en distintas empresas, se incrementaron los niveles de exposición a riesgos psicosociales, físicos y ergonómicos (Vicente-Herrero et al., 2018). Los riesgos surgen a raíz del uso excesivo de las tecnologías de la información y comunicación, sumado a las condiciones físicas y ambientales en las que el trabajador desempeña sus actividades diarias (Cardenas-Velasquez \& Bracho-Paz, 2020).

El uso del computador, sus periféricos, el apoyo de las muñeca y la postura incorrecta de la columna vertebral, son elementos que pueden causar lesiones osteomioarticulares en los docentes que realizan actividades de teletrabajo, lo cual predispone al docente a disminuir el uso de estas tecnologías y como consecuencia generar aversión a esta modalidad (Tapasco Alzate \& Giraldo García, 2016), por esta razón es necesario que los equipos, como sillas, escritorios y periféricos sean seleccionados acorde a las características físicas del tele trabajador para minimizar los problemas de salud laboral (Alcón, 2020).

Cabe recalcar que se observa el aparecimiento de trastornos OMA en tele trabajadores, es por ello que se han planteado métodos que se basen en la correcta postura corporal (García-Salirrosas EE \& Sánchez-Poma RA, 2020), desde la década de los 70 se tiene registros de métodos para evaluar posturas de los trabajadores sobre todo en puestos de oficina dando buenos resultados el método ROSA. Esta investigación considera el estudio 
ergonómico global por posturas de trabajo, importante para disminuir el riesgo ergonómico de los trabajadores (García Becerra et al., 2018).

La postura correcta de espalda, brazos y piernas para atenuar el nivel de riesgo ergonómico por teletrabajo necesita de un cambio en la posición del ocupante moviendo el plano de trabajo de las sillas donde realiza la mayor parte de sus actividades (Sánchez, 2018) buscando conseguir alinear la espalda con el eje de cadera y piernas, también buscando la posición de los brazos por debajo del nivel de los hombros, y las piernas debido al peso del cuerpo deberían descansar mayoritariamente sobre los isquiones de la persona (Larrea-Araujo et al., 2021)

Por otro lado, la afectación directa del uso de sillas disergonómicas se pudo evidenciar con el cuestionario nórdico para determinar síntomas OMA con la aplicación de un instrumento con preguntas relacionadas a síntomas de molestias osteomioarticulares (González Muñoz, 2021).

Este trabajo aplica una medida ergonómica correctiva en los ejes ajustables de las sillas tipo altura, longitud, respaldo, reposabrazos desarrollado para los docentes de la comisión técnica del área de contabilidad de la UEHA. Para evidenciar la mejora se utilizó el método ROSA previo y una evaluación con las mejoras aplicadas (López \& Franco, 2019).

\section{Metodología}

Se ha realizado una investigación prospectiva, correlacional, cuali cuantitativa. Se evaluó a los docentes de la Unidad Educativa Hispano América en la modalidad de teletrabajo, para lo cual se aplicó una hoja de campo de Ergosoft, para la implementación y evaluación del Método ROSA relacionado con la silla, pantalla y periféricos que utiliza el trabajador en sus actividades; siendo aplicable para las personas que permanecen sentados en una silla, frente a un computador, a fin de precautelar la seguridad y salud del tele trabajador (Torres et al., 2020).

\section{Cuestionario Nórdico.}

La constante evolución tecnológica ha permitido el diseño y validación de numerosos instrumentos que permiten realizar una evaluación adecuada de las actividades laborales (Angarita et al., 2020). Para la realización de la presente investigación se ha tomado en cuenta como instrumento de evaluación al cuestionario Nórdico, diseñado y validado para la investigación de trastornos y afecciones OMA, que a pesar de no ser el único instrumento aplicable en este tipo de estudios, se considera el más apropiado por la información que aporta en relación a la aparición de sintomatología, duración y manejo del cuadro (Martínez \& Alvarado Muñoz, 2017).

Esta herramienta fue publicada en 1987 y es una de las más utilizadas para la evaluación de trastornos OMA a nivel laboral, permitiendo obtener resultados fiables y confiables (Kuorinka et al., 1987). Se compone de dos secciones importantes, la primera que se enfoca en la sintomatología referida por el trabajador y el área corporal afectada; y por 
otro lado, en la segunda parte se indaga a profundidad la duración de la sintomatología, el seguimiento que se ha realizado y la evaluación médica (García-Salirrosas EE \& Sánchez-Poma RA, 2020).

Tabla 1.

Parámetros de evaluación del Cuestionario Nórdico

\begin{tabular}{ll}
\hline \multicolumn{1}{c}{ Método } & \multicolumn{1}{c}{ Puntos de evaluación } \\
\hline Cuestionario & a) Localización anatómica de la molestia. \\
Nórdico & b) Tiempo de evolución de la molestia. \\
& c) Necesidad de cambio de puesto de trabajo. \\
& d) Molestias en el último año. \\
& e) Duración de la molestia durante el último año. \\
& f) Duración de cada episodio. \\
g) Limitación de actividad laboral. \\
h) Antecedentes de tratamiento. \\
i) Molestias en los últimos 7 días. \\
j) Valoración numérica de las molestias. \\
k) Etiología de las molestias.
\end{tabular}

Nota: Los puntos de evaluación del cuestionario Nórdico se enfocan en la localización, duración, etiología y manejo de las molestias OMA.

Fuente: (Kuorinka et al., 1987)

\section{Método ROSA}

En la actualidad, la mayor parte de puestos de trabajo incluyen en sus labores diarias el uso de pantallas de visualización de datos, lo que conlleva a la necesidad de que el trabajador permanezca sentado por largos períodos de tiempo, especialmente en puestos de oficina (Diego-Mas, 2015). Esta situación ha incrementado los trastornos OMA en los trabajadores, con énfasis en miembros superiores, cuello y espalda (Real \& Cedeño, 2020). El método Rapid Office Strain Assessment (ROSA) es considerado una herramienta indispensable para la evaluación de los puestos de trabajo en oficina. Incluye la evaluación de la silla, pantalla, mouse, teléfono, entre otros, de los que se obtienen valores cuantitativos que permiten determinar la necesidad de mejora (Diego-Mas, 2015).

El proceso de evaluación incluye asignar un puntaje a cada elemento del puesto de trabajo luego de observar al trabajador durante su jornada y aplicando una hoja de campo para el método (Real \& Cedeño, 2020). Con la puntuación asignada, se obtendrán resultados parciales y finales para determinar el nivel de actuación que se debe tomar en cuenta y las medidas que se deben corregir. Los valores de la puntuación ROSA pueden ir de 1 a 10, donde a mayor valor existe mayor riesgo para el trabajador del puesto estudiado (Diego-Mas, 2015).

Tabla 2.

Riesgo y niveles de actuación ROSA

\begin{tabular}{cccl}
\hline Puntuación & Riesgo & Nivel & Actuación \\
\hline $\mathbf{1}$ & Inapreciable & 0 & No es necesaria actuación. \\
\hline $\mathbf{2}$ - $\mathbf{3}-\mathbf{4}$ & Mejorable & 1 & Pueden mejorarse algunos elementos del puesto. \\
\hline
\end{tabular}


Tabla 2.

Riesgo y niveles de actuación ROSA (continuación)

\begin{tabular}{cccl}
\hline Puntuación & Riesgo & Nivel & \multicolumn{1}{c}{ Actuación } \\
\hline $\mathbf{5}$ & Alto & 2 & Es necesaria la actuación. \\
\hline $\mathbf{6 - 7}-\mathbf{8}$ & Muy Alto & 3 & Es necesaria la actuación cuanto antes. \\
\hline $\mathbf{9 - 1 0}$ & Extremo & 4 & Es necesaria la actuación urgentemente.
\end{tabular}

Nota: Los niveles de actuación determinan la necesidad de actuación en el puesto de trabajo, en base a 1 puntuación final obtenida en el método ROSA.

Fuente: (Diego-Mas, 2015)

\section{Proceso de evaluación del método ROSA}

El primer elemento que evalúa el método ROSA es la silla de trabajo, a la cual se le asignan valores cuantitativos en base a la observación de: Altura y profundidad del asiento, reposabrazos y respaldo, con puntajes que oscilan entre 1, 2 y 3 puntos y variación de puntuación en situaciones especiales. Una vez obtenidas las puntuaciones se suma la Altura del Asiento y la Profundidad del Asiento, y por otro lado los puntajes de los Reposabrazos y el Respaldo para obtener la puntuación A, a la que se sumará la puntuación de acuerdo al tiempo de uso de la silla. (Diego-Mas, 2015).

Tras haber obtenido la puntuación de la silla se procederá a obtener la de la Pantalla y periféricos (teclado, mouse y teléfono), asignando un valor a cada uno de ellos en base a la observación previa y sumando a cada medición el puntaje correspondiente al tiempo de uso durante la jornada laboral para obtener las puntuaciones parciales. Se procede a sumar la pantalla y el teléfono, y por otro lado el mouse y el teclado para obtener los valores de las tablas B y C, con las que se obtendrá el valor de la tabla $\mathrm{D}$, mismo que al ser conjugado con la puntuación de la silla, brindará la puntuación final del método ROSA y por ende contribuirá a determinar el nivel de actuación para el puesto de trabajo (DiegoMas, 2015).

\section{Resultados y discusión}

\section{Resultados del Cuestionario Nórdico}

Con respecto a los resultados del cuestionario aplicado, se obtuvo que de los 12 docentes del Área de la Comisión Técnica de Contabilidad evaluados, han presentado en su totalidad algún tipo de molestias OMA, el 75\% a nivel de región lumbar, 50\% a nivel de mano y muñeca, $41,67 \%$ a nivel cervical, $33,33 \%$ a nivel de hombro y el $16,67 \%$ a nivel de codo y antebrazo (Tabla 3). Sobre la duración de la sintomatología presentada, manifestaron que las molestias a nivel lumbar permanecían por 7días, con un tiempo de duración de cada episodio de 2 horas que cede con analgésicos por vía oral y en ocasiones administración intramuscular. 
Tabla 3.

Resultados evaluativos del Cuestionario Nórdico

\begin{tabular}{lcc}
\hline \multicolumn{1}{c}{ MOLESTIAS } & RESULTADOS EVALUACION* & PORCENTAJE \\
\hline Cuello & 5 de 12 trabajadores evaluados & $41,67 \%$ \\
\hline Hombro & 4 de 12 trabajadores evaluados & $33,33 \%$ \\
\hline Dorsal o lumbar & 9 de 12 trabajadores evaluados & $75 \%$ \\
\hline Codo o antebrazo & 2 de 12 trabajadores evaluados & $16,67 \%$ \\
\hline Muñeca o mano & 6 de 12 trabajadores evaluados & $50 \%$ \\
\hline
\end{tabular}

Nota: *Los resultados de evaluación mostrados en la tabla constituyen las respuestas afirmativas que se obtuvieron de los 12 docentes evaluados, de los cuales cada uno de ellos señaló las localizaciones anatómicas que les causaban molestias

Fuente: (Kuorinka et al., 1987)

\section{Resultados del método ROSA previo y ROSA con mejoras del puesto de trabajo}

A continuación, se evidencian los resultados del análisis de carga postural estática adoptada por los docentes.

Tabla 4.

Resultados evaluativos del método ROSA previo vs. ROSA con mejoras del puesto de trabajo

\section{Cálculo de la puntuación ROSA previo}

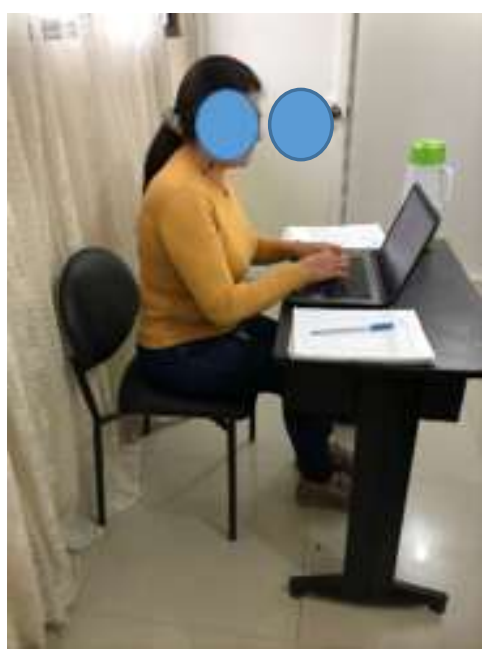

\begin{tabular}{l|l|l|l|c|c|c|c|c}
\hline \multicolumn{4}{c}{ Silla } & $\begin{array}{l}\text { Monito } \\
\mathrm{r}\end{array}$ & $\begin{array}{l}\text { Teléfon } \\
\mathrm{o}\end{array}$ & $\begin{array}{l}\text { Teclad } \\
\mathrm{o}\end{array}$ & $\begin{array}{l}\text { Rató } \\
\mathrm{n}\end{array}$ \\
\hline $\begin{array}{l}\text { Altur } \\
\text { a }\end{array}$ & $\begin{array}{l}\text { Longitu } \\
\mathrm{d}\end{array}$ & $\begin{array}{l}\text { Reposabraz } \\
\text { os }\end{array}$ & $\begin{array}{l}\text { Respald } \\
\mathrm{o}\end{array}$ & $\begin{array}{l}\text { Tota } \\
1\end{array}$ & & & & \\
\hline 3 & 3 & 3 & 3 & 7 & 4 & 0 & 3 & 2 \\
\hline
\end{tabular}


Tabla 4.

Resultados evaluativos del método ROSA previo vs. ROSA con mejoras del puesto de trabajo (continuación)

\section{Cálculo de la puntuación ROSA con mejoras del puesto de trabajo}



\begin{tabular}{c|c|c|c|c|c|c|c|c}
\hline \multicolumn{4}{c}{ Silla } & $\begin{array}{c}\text { Monito } \\
\mathrm{r}\end{array}$ & $\begin{array}{c}\text { Teléfon } \\
\mathrm{o}\end{array}$ & $\begin{array}{c}\text { Teclad } \\
\mathrm{o}\end{array}$ & $\begin{array}{c}\text { Rató } \\
\mathrm{n}\end{array}$ \\
\hline $\begin{array}{c}\text { Altur } \\
\text { a }\end{array}$ & $\begin{array}{c}\text { Longitu } \\
\mathrm{d}\end{array}$ & $\begin{array}{c}\text { Reposabraz } \\
\text { os }\end{array}$ & $\begin{array}{c}\text { Respald } \\
\text { o }\end{array}$ & $\begin{array}{c}\text { Tota } \\
1\end{array}$ & & & & \\
\hline 1 & 3 & 2 & 1 & 4 & 3 & 0 & 2 & 2 \\
\hline
\end{tabular}

Nota: Evaluación inicial y evaluación con la inclusión de silla ajustable en el puesto de trabajo

Fuente: (Diego-Mas, 2015)

El método ROSA previo revela una puntuación final de 7 en una escala del 1 al 10, lo cual corresponde a un nivel de riesgo 3 (Muy alto), que se correlaciona con la necesidad de actuación cuanto antes para mejorar el puesto de trabajo y disminuir el riesgo de los trabajadores. Por ello se decide la inclusión de sillas ajustables en el ambiente laboral del docente tele trabajador, consiguiendo mejorar la puntuación final a 3 (Mejorable), que corresponde a nivel de riesgo 1 y en el cual los trabajadores se beneficiarán drásticamente de las mejoras implementadas con respecto a los trastornos osteomioarticulares que han venido presentando.

\section{Mejora de la Silla.}

La silla ha resultado ser uno de los elementos con mayor puntaje en la evaluación, debido a que los docentes del estudio permanecen más del tiempo recomendado en posición de sedestación, sobrepasando las 4 horas de jornada laboral, lo cual es muy difícilmente modificable, sin embargo se pueden implementar pausas activas periódicas, al menos cada hora durante la jornada. En la mejora implementada sea regulado el asiento de la silla, ajustándolo a una altura que permita que el ángulo de flexión de los miembros inferiores del trabajador sea del $90^{\circ}$ con los pies apoyados en el piso, para prevenir la 
presión excesiva en región glútea y columna vertebral. Por otro lado, se ha logrado respetar los $8 \mathrm{~cm}$ aproximados que deben existir entre el borde del asiento y la región poplítea posterior. Por último y una parte muy importante constituye el respaldo de la silla, el cual debe brindar apoyo a la región lumbar del trabajador, respetando la curvatura natural.

\section{Mejora de pantalla y periféricos}

Al igual que con la silla, es imprescindible que si no se puede reducir el tiempo de uso de pantalla de visualización de datos y periféricos, por lo menos se integre a la jornada laboral pausas activas periódicas, de al menos cada hora. De igual manera, es necesario el ajuste de la pantalla en altura, procurando que no sea demasiado alta o demasiado baja, debiendo localizarse a nivel de los ojos del trabajador con el fin de no ocasionar lesiones cervicales. De igual manera, se debe escoger el tamaño de mouse adecuado para cada trabajador, con el fin de evitar posturas inadecuadas de la mano al utilizarlo, localizándolo al mismo nivel del teclado.

Tabla 5.

Puntuaciones asignadas a la silla en el método ROSA previo vs. ROSA con mejoras.

\begin{tabular}{|c|c|c|c|c|}
\hline \multicolumn{3}{|c|}{ SILLA } & $\begin{array}{l}\text { ROSA } \\
\text { previo }\end{array}$ & $\begin{array}{c}\text { ROSA } \\
\text { con } \\
\text { mejoras }\end{array}$ \\
\hline \multicolumn{2}{|c|}{ Altura silla } & Puntos & & \multirow{5}{*}{1} \\
\hline \multirow{4}{*}{$\begin{array}{l}\text { Altura no ajustable } \\
+1 \\
\text { Sin suficiente } \\
\text { espacio bajo la } \\
\text { mesa: }+1\end{array}$} & Rodillas a $90^{\circ}$ & 1 & \multirow[t]{4}{*}{$2+1$} & \\
\hline & Silla baja. Rodillas $<90^{\circ}$ & 2 & & \\
\hline & Silla alta. Rodillas $>90^{\circ}$ & 2 & & \\
\hline & Sin contacto con el suelo & 3 & & \\
\hline \multicolumn{2}{|c|}{$\begin{array}{l}\text { Longitud del asiento } \\
\end{array}$} & Puntos & \multirow{4}{*}{$2+1$} & \multirow{4}{*}{$2+1$} \\
\hline \multirow[t]{3}{*}{$\begin{array}{l}\text { Longitud no } \\
\text { ajustable: }+1\end{array}$} & $\begin{array}{l}\mathrm{cm} \text {. Entre borde de silla y } \\
\text { rodilla }\end{array}$ & 1 & & \\
\hline & $\begin{array}{l}\text { Menos de } 8 \mathrm{~cm} \text { de entre el } \\
\text { borde de la silla y la rodilla }\end{array}$ & 2 & & \\
\hline & $\begin{array}{l}\text { Más de } 8 \mathrm{~cm} \text { entre el borde } \\
\text { de la silla y la rodilla }\end{array}$ & 2 & & \\
\hline \multicolumn{2}{|c|}{ Reposabrazos } & Puntos & \multirow{3}{*}{$2+1$} & \multirow{3}{*}{$1+1$} \\
\hline \multirow{3}{*}{$\begin{array}{l}\text { Brazos separados: } \\
+1 \\
\text { Reposabrazos duro } \\
\text { o dañado: }+1 \\
\text { No ajustable: }+1 \\
\end{array}$} & En línea. Hombro relajado. & 1 & & \\
\hline & $\begin{array}{l}\text { Muy alto o con poco } \\
\text { soporte }\end{array}$ & 2 & & \\
\hline & Respaldo & Puntos & & \\
\hline \multirow{3}{*}{$\begin{array}{l}\text { No ajustable: }+1 \\
\text { Mesa de trabajo } \\
\text { muy alta: }+1\end{array}$} & Respaldo recto y ajustado & 1 & \multirow[t]{3}{*}{$2+1$} & \multirow[t]{3}{*}{1} \\
\hline & $\begin{array}{l}\text { Respaldo pequeño y sin } \\
\text { apoyo }\end{array}$ & 2 & & \\
\hline & $\begin{array}{l}\text { Respaldo demasiado } \\
\text { inclinado }\end{array}$ & 2 & & \\
\hline
\end{tabular}


Tabla 5.

Puntuaciones asignadas a la silla en el método ROSA previo vs. ROSA con mejoras.

\begin{tabular}{|c|c|c|c|c|}
\hline \multicolumn{3}{|c|}{ SILLA } & \multirow[t]{2}{*}{$\begin{array}{l}\text { ROSA } \\
\text { previo }\end{array}$} & \multirow[t]{2}{*}{$\begin{array}{l}\text { ROSA } \\
\text { con } \\
\text { mejoras } \\
\end{array}$} \\
\hline & Respaldo & Puntos & & \\
\hline $\begin{array}{l}\text { No ajustable: }+1 \\
\text { Mesa de trabajo } \\
\text { muy alta: }+1\end{array}$ & $\begin{array}{l}\text { Inclinado y espalda sin } \\
\text { apoyar }\end{array}$ & 2 & & \\
\hline \multicolumn{2}{|r|}{ Duración } & Puntos & \multirow{4}{*}{+1} & \multirow{4}{*}{+1} \\
\hline$<1$ hora/día o $<$ & ainutos seguidos & -1 & & \\
\hline 1-4 hora/día o 3 & in - $1 \mathrm{~h} /$ continuado & 0 & & \\
\hline$>4$ horas/día o > & ora continuado & +1 & & \\
\hline
\end{tabular}

Nota: El ROSA con mejoras muestra los resultados con la inclusión de sillas de trabajo ajustables en los docentes que realizan teletrabajo.

Fuente: (Diego-Mas, 2015)

Tabla 6.

Puntuaciones asignadas al Monitor y Periféricos en el método ROSA previo vs. ROSA con mejoras

\begin{tabular}{|c|c|c|c|c|}
\hline \multicolumn{3}{|c|}{ Monitor y periféricos } & $\begin{array}{l}\text { ROSA } \\
\text { previo }\end{array}$ & $\begin{array}{c}\text { ROSA } \\
\text { con } \\
\text { mejoras }\end{array}$ \\
\hline \multicolumn{2}{|c|}{ Monitor } & Puntos & & \multirow{4}{*}{$1+1$} \\
\hline \multirow{3}{*}{$\begin{array}{l}\text { Monitor muy lejos: }+1 \\
\text { Reflejos en monitor: }+1 \\
\text { Documentos sin } \\
\text { soporte: }+1 \\
\text { Cuello girado: }+1\end{array}$} & $\begin{array}{l}\text { Posición ideal, parte } \\
\text { superior a la altura de } \\
\text { los ojos }\end{array}$ & 1 & \multirow[t]{3}{*}{$2+1$} & \\
\hline & Monitor bajo. & 2 & & \\
\hline & Monitor alto. & 3 & & \\
\hline \multicolumn{2}{|c|}{ Duración } & Puntos & & \\
\hline \multicolumn{2}{|c|}{$<1$ hora/día o $<30$ minutos seguidos } & -1 & \multirow[t]{3}{*}{+1} & \multirow[t]{3}{*}{+1} \\
\hline \multicolumn{2}{|c|}{ 1-4 hora/día o $30 \mathrm{~min}$ - 1h/continuado } & 0 & & \\
\hline \multicolumn{2}{|c|}{$>4$ horas/día o > 1hora continuado } & +1 & & \\
\hline \multicolumn{2}{|c|}{ Teléfono } & Puntos & & \\
\hline \multirow{2}{*}{$\begin{array}{l}\text { Teléfono en cuello y } \\
\text { hombro: }+2 \\
\text { Sin manos libres: }+1\end{array}$} & $\begin{array}{l}\text { Teléfono una mano o } \\
\text { manos libres }\end{array}$ & 1 & \multirow[t]{2}{*}{1} & \multirow[t]{2}{*}{1} \\
\hline & Teléfono muy alejado & 2 & & \\
\hline \multicolumn{2}{|c|}{ Duración } & Puntos & & \\
\hline$<1$ hora/día o $<30 \mathrm{minu}$ & os seguidos & -1 & \multirow[t]{3}{*}{-1} & \multirow[t]{3}{*}{-1} \\
\hline 1-4 hora/día o $30 \mathrm{~min}$ - & h/continuado & 0 & & \\
\hline \multirow{2}{*}{\multicolumn{2}{|c|}{$\begin{array}{c}>4 \text { horas/día o }>\text { 1hora continuado } \\
\text { Teclado }\end{array}$}} & +1 & & \\
\hline & & Puntos & & \\
\hline \multirow{2}{*}{$\begin{array}{l}\text { Muñecas desviadas: }+1 \\
\text { Teclado muy alto: }+1 \\
\text { Objetos por encima de } \\
\text { la cabeza: }+1 \\
\text { No ajustable: }+1 \\
\end{array}$} & $\begin{array}{l}\text { Muñecas rectas } \\
\text { hombros relajados }\end{array}$ & 1 & \multirow[t]{2}{*}{$1+1$} & \multirow[t]{2}{*}{1} \\
\hline & $\begin{array}{l}\text { Muñecas extendidas } \\
\text { más de } 15^{\circ}\end{array}$ & 2 & & \\
\hline \multicolumn{2}{|c|}{ Duración } & Puntos & & \\
\hline \multicolumn{2}{|c|}{$<1$ hora/día o $<30$ minutos seguidos } & -1 & +1 & +1 \\
\hline
\end{tabular}


Tabla 6.

Puntuaciones asignadas al Monitor y Periféricos en el método ROSA previo vs. ROSA con mejoras (continuación)

\begin{tabular}{|c|c|c|c|c|}
\hline \multicolumn{3}{|c|}{ Monitor y periféricos } & $\begin{array}{l}\text { ROSA } \\
\text { previo }\end{array}$ & $\begin{array}{c}\text { ROSA } \\
\text { con } \\
\text { mejoras }\end{array}$ \\
\hline \multicolumn{2}{|c|}{ Duración } & Puntos & & \\
\hline \multicolumn{2}{|c|}{ 1-4 hora/día o $30 \mathrm{~min}$ - 1h/continuado } & 0 & & \\
\hline \multicolumn{2}{|c|}{$>4$ horas/día o > 1hora continuado } & +1 & & \\
\hline \multicolumn{2}{|c|}{ Ratón } & Puntos & & \\
\hline \multirow{2}{*}{$\begin{array}{l}\text { Ratón y teclado en } \\
\text { diferentes alturas: }+2 \\
\text { Ratón pequeño: }+1 \\
\text { Reposamanos delante } \\
\text { del ratón: }+1\end{array}$} & $\begin{array}{l}\text { Ratón en línea con el } \\
\text { hombro }\end{array}$ & 1 & 1 & 1 \\
\hline & $\begin{array}{l}\text { Ratón con brazo lejos } \\
\text { del cuerpo }\end{array}$ & 2 & & \\
\hline \multicolumn{2}{|c|}{ Duración } & Puntos & & \\
\hline \multicolumn{2}{|c|}{$<1$ hora/día o $<30$ minutos seguidos } & -1 & +1 & +1 \\
\hline \multicolumn{2}{|c|}{ 1-4 hora/día o $30 \mathrm{~min}$ - 1h/continuado } & 0 & & \\
\hline \multicolumn{2}{|c|}{$>4$ horas/día o > 1hora continuado } & +1 & & \\
\hline
\end{tabular}

Nota: El método ROSA con mejoras muestra los resultados con la inclusión de la silla ajustable al ambiente de trabajo del docente

Fuente: (Diego-Mas, 2015)

\section{Conclusiones}

- El presente estudio se ha aplicado a 12 docentes de la Comisión Técnica del Área de Contabilidad de la Unidad Educativa Hispano América que realizan teletrabajo y han presentado molestias osteomioarticulares, identificadas mediante la aplicación del cuestionario nórdico, obteniéndose resultados de que las lesiones más frecuentes se encuentran a nivel de región lumbar; posterior a lo cual se aplicó el método ROSA, que en la evaluación preliminar reportó un resultado de 7 , correspondiente a un riesgo muy alto y con necesidad de intervención del puesto de trabajo; para lo cual se plantearon mejoras a nivel de la silla que utilizan los docentes durante el teletrabajo, con lo cual se mejoró la puntuación final del método ROSA, pasando a 4 puntos, que corresponde a riesgo nivel 1 (mejorable), lo cual al ser incorporado en todos los docentes disminuirá significativamente las molestias lumbares OMA que se han venido presentando durante el período de evaluación.

\section{Referencias bibliográficas.}

Alcón, P. G. (2020). El teletrabajo como estrategia empresarial sostenible en una empresa de servicios de consultoría TT - Teleworking as a sustainable business strategy in a consulting services company. Revista Ibérica de Sistemas e Tecnologias de Informação, E31, 390-403.

Angarita, K. E., Gaona-García, P., Garzón, R. C., Gómez, D. N., \& Montenegro-Marín, 
C. (2020). ErgoSent: Prototipo informático como soporte a la evaluación ergonómica de trabajadores sobre el área de marketing y diseño TT - ErgoSent: Computer prototype as support for office ergonomics. Revista Ibérica de Sistemas e Tecnologias de Informação, E35, 246-262.

Benites-Morillas, H. A., Rojas-Ciudad, C. A., Vásquez-Pereyra, Y. Y., \& PuentesAzabache, G. R. (2021). Ergonomía y la práctica docente en el contexto remoto. Revista Científica Dominio de Las Ciencias, 7(3), 41-60.

Cardenas-Velasquez, A. J., \& Bracho-Paz, D. C. (2020). El Tecnoestrés: Una consecuencia de la inclusión de las TIC en el trabajo. Cienciamatria, 6(1), 295-314.

Chuco Aguilar, V. J. (2021). El teletrabajo y su impacto en el estrés de los trabajadores. Neumann Business Review, 7(1), 81-98. https://doi.org/10.22451/3006.nbr2021.vol7.1.10059

Diego-Mas, J. A. (2015). Evaluación de puestos de trabajo de oficinas mediante el método ROSA. Ergonautas. http://www.ergonautas.upv.es/metodos/rosa/rosaayuda.php

García-Salirrosas EE, \& Sánchez-Poma RA. (2020). Prevalencia de trastornos musculoesqueléticos en docentes universitarios que realizan teletrabajo en tiempos de COVID-19. An Fac Med., 81(3), 301-307.

García Becerra, A., Cano Gutiérrez, J. C., Ensaldo Rentería, E. V., Camargo Wilson, C., Olguín Tiznado, J. E., \& López Barreras, J. A. (2018). Evaluación Ergonómica En El Módulo De Préstamos De Una Biblioteca De Universidad Pública. Revista Ingenieria Industrial, 17(2), 171-186. https://doi.org/10.22320/s07179103/2018.10

González Muñoz, E. L. (2021). Estudio de Validez y confiabilidad del Cuestionario Nórdico Estandarizadopara detección de síntomas musculoesqueléticos en Población Mexicana. Ergonomía, Investigación y Desarrollo, 3(1), 8-17.

Kuorinka, I., Jonsson, B., Kilbom, A., Vinterberg, H., Biering-Sørensen, F., Andersson, G., \& Jørgensen, K. (1987). Standardised Nordic questionnaires for the analysis of musculoskeletal symptoms. Applied Ergonomics, 18(3), 233-237.

Larrea-Araujo, C., Ayala-Granja, J., Vinueza-Cabezas, A., \& Acosta-Vargas, P. (2021). Ergonomic risk factors of teleworking in ecuador during the covid-19 pandemic: A cross-sectional study. International Journal of Environmental Research and Public Health, 18(10).

López, S., \& Franco, D. (2019). Factor de riesgo ergonómico por videoterminal en teletrabajadores. Perspectivas En Inteligencia, 11(20), 335-346.

Martínez, M. M., \& Alvarado Muñoz, R. (2017). Validación del Cuestionario Nórdico Estandarizado de Síntomas Musculoesqueléticos para la población trabajadora chilena, adicionando una escala de dolor. Revista de Salud Pública, 21(2), 43. 
Montero Ulate, B., Vasconcelos Vásquez, K. L., \& Arias Murillo, G. (2020). Teletrabajo: fortaleciendo el trabajo en tiempos de pandemia por COVID-19. Revista de $\begin{array}{lllll}\text { Comunicación } & y & \text { Salud, } & 10(2), & 109-125 .\end{array}$ https://doi.org/10.35669/rcys.2020.10(2).109-125

Real, G., \& Cedeño, L. (2020). Procedimiento para la evaluación de los factores de riesgo laboral y su incidencia en el desempeño laboral en usuarios de Pantallas de Visualización de Datos (PVD). Ingeniería Industrial, 039, 15-34. https://doi.org/10.26439/ing.ind2020.n039.4913

Sánchez, A. (2018). Prevalencia de desórdenes músculo esqueléticos en trabajadores de una empresa de comercio de productos farmacéuticos. Revista Ciencias de La Salud, 16(2), 203.

Tapasco Alzate, O., \& Giraldo García, J. (2016). Factores asociados a la disposición por el teletrabajo entre docentes universitarios. Ciencia \& Trabajo, 18(56), 87-93.

Torres, S. J., Paladines, C. A., Luzuriaga, W. D., \& Cabezas, E. B. (2020). Diseño de estación de telestudio ergonómica para mejora postural en alumnos de posgrado de la Universidad Técnica Particular de Loja. Revista Espacios, 41(35), 15. http://www.revistaespacios.com/a20v41n35/a20v41n35p10.pdf

Vallejo Morán, J. C., Bustillos Molina, I. T., Martínez Porro, E., \& Coello Leon, E. C. (2021). Evaluación ergonómica mediante el método ROSA en docentes con teletrabajo de la UTEQ, 2020. Ingeniería e Innovación, 8(22). https://doi.org/10.21897/23460466.2330

Vicente-Herrero, M. T., Torres Alberich, J. I., Torres Vicente, A., Ramirez, M. V., \& Capdevila Garcia, L. (2018). El teletrabajo en salud laboral. Revista Ces Derecho, 9(2), 287-297. 


\section{PARA CITAR EL ARTÍCULO INDEXADO.}

Vargas Arboleda, P. E., \& Córdova Suárez, M. A. (2021). Uso de sillas ajustables en docentes que realizan teletrabajo y presentan molestias osteomioarticulares en la Unidad Educativa Hispano América. Anatomía Digital, 4(3.1), 63-76. https://doi.org/10.33262/anatomiadigital.v4i3.1.1861

\section{LCiencia}

El artículo que se publica es de exclusiva responsabilidad de los autores y no necesariamente reflejan el pensamiento de la Revista Anatomía Digital.

El artículo queda en propiedad de la revista y, por tanto, su publicación parcial y/o total en otro medio tiene que ser autorizado por el director de la Revista Anatomía Digital.
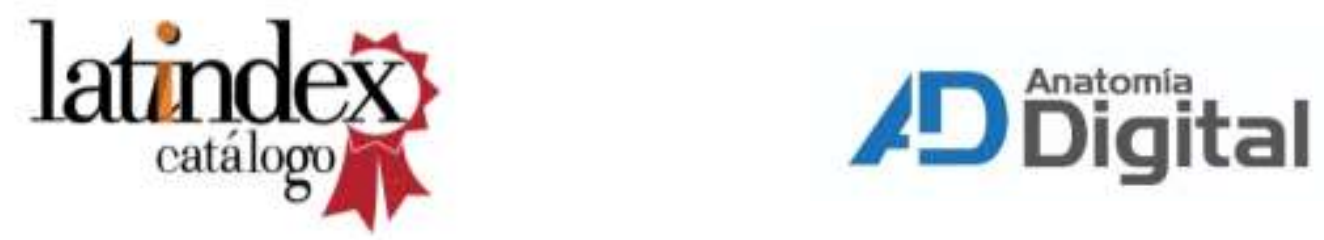